\title{
White Paper: Improving Handoff Culture in Intensive Care Unit to Floor Handoffs
}

\author{
Grant Turner, MD, Kristin Lohr, MD, Andrew Brown, MD, Allison Greco, MD, and Rebecca Jaffe, MD
}

\begin{abstract}
The frequency of handoffs between providers has increased since the 2011 Accreditation Council for Graduate Medical Education (ACGME) work hour restrictions, generating concerns over the quality of these handoffs and their impact on patient safety. At Thomas Jefferson University Hospital (TJUH), the 2016 Safety Culture Survey revealed that across all specialties, many residents felt that "things fall through the cracks" when transferring patients from one unit to another. The interdepartmental Housestaff Quality and Safety Leadership Council (HQSLC) at TJUH sought to improve handoffs at our institution and identified two areas of focus: (1) standardizing the language of handoffs with a commonly accepted handoff technique (IPASS), and (2) standardizing the process of handoffs from the ICU to the floor. Qualitatively, resident comfort with handoffs improved with no adverse impact on time to patient movement between units. This project demonstrated the difficulty of changing the handoff culture at an institution, establishing lasting change via a new EMR system, and training housestaff of a new handoff method. Future directions include monitoring compliance with the new standardized handoff curriculum, and determining whether these efforts and interventions translate to improved patient safety at our institution.
\end{abstract}

\section{BACKGROUND}

The frequency of handoffs between providers has increased following the implementation of the ACGME work hour restrictions. In this context, properly structured and timed handoffs are essential to patient safety now more than ever. ${ }^{1}$ Despite this, studies have shown that errors in communication of code status, medication allergies, and changes to plan of care are common; errors which can lead to adverse outcomes to patients. ${ }^{2}$ Improving the quality of handoffs between providers is a growing priority in an effort to reduce medical errors. In alignment with these goals, the standardized handoff curriculum known as I-PASS, a mnemonic for "illness severity, patient summary, action list, situation awareness, and synthesis by receiver" has been validated in single-center and then multi-center trials which showed a reduction in medical error rate by $23 \%$ and the rate of preventable adverse events by $30 \% .3 .4$
At TJUH, the 2016 Agency for Healthcare Research and Quality Hospital Survey on Patient Safety Culture (AHRQ HSOPS) was administered to 869 house staff. Of 639 respondents, only $43 \%$ viewed handoffs and transitions favorably across all specialties. $37 \%$ of residents felt that "things fall through the cracks" when transferring patients from one unit to another. The ACGME's Clinical Learning Environment Review (CLER) committee regularly reviews the culture of handoffs at training programs and had also identified this as an area for improvement in their 2015 report to TJUH, specifically identifying ICU to floor handoffs as an area of weakness.

In this context, the HQSLC, a group comprised of 30 house staff from 15 departments which seeks to strategically impact key quality and safety issues across the institution, chose to focus their annual project on improving transitions of care at TJUH. This interdepartmental working group identified the following root causes affecting the safety of handoffs and transitions of care at TJUH: 1) lack of standardization in handoff content and transfer process, (2) variation in handoff training curricula between training programs, and (3) failure of the former electronic health record to reinforce best practices or ideal processes. To target these root causes, a specific focus was put on standardizing house staff handoff practice using the ICU to floor transfer as a prototype, as well as standardizing the content of handoffs used by house staff across the institution through implementation of the IPASS handoff curriculum.

\section{INTERVENTION}

An ideal handoff should be safe, timely, effective, efficient, equitable and patient-centered (STEEEP). ${ }^{5}$ With this in mind, we proposed the following 4-tiered model to implement change to the current house staff training and practice of handoffs at TJUH:

1. Build Momentum: Identify department-based champions, to include a resident and faculty member dyad.

2. Standardize Curricula: Create a training module for use in Jefferson GME programs, based on the framework described by the I-PASS study group, and incorporating both didactics and simulation exercises facilitated by resident peers. 
3. Assess Proficiency: Develop a framework to assess learner mastery and provide longitudinal feedback regarding performance.

4. Reinforce Compliance: Best practices should be made easy to follow through intentional process design, health IT, innovative team structures, and feedback to frontline providers.

In order to support step 1 of the model, a faculty member in every core GME training program was selected to participate in TeamSTEPPS Master Training.

Evaluation of the existing workflow for ICU to floor handoffs at TJUH identified barriers to safe transfers, as well as existing processes that could be harnessed to reinforce high reliability and safe communication. A high degree of variation in the ICU to floor transfer process was found in the following areas: (1) timing of the handoff, (2) incorporation of best practice of using both a verbal and written handoff, and (3) use of closed loop communication between sending and receiving teams.

The workgroup determined an ideal workflow for a safe ICU to floor transfer should include: (1) bedside evaluation by the ICU team and documentation of this in the medical record prior to transfer, followed by (2) a verbal and written handoff between sending and receiving residents, and finally (3) review of patient orders and placement of an order (physician staff information) signifying that a handoff had occurred and identifying the new primary team. To improve compliance with this new process, a new hard stop was created in the transfer process restricting a patient from moving to the new unit until the order review and handoff was completed by house staff. Residents staffing the ICU as well as nursing and physician leadership in the ICU were educated on the intervention and process change prior to its implementation. Residents were surveyed at the end of each month of the pilot to determine barriers to behavior change.

To evaluate compliance with the process change, chart review was done to determine frequency of the bedside evaluation and ensure the order for team staff information change was placed by a member of the receiving team. Data from the patient flow management center on time from assignment of floor bed to transfer out of the ICU was also analyzed to evaluate effects on total transfer time.

\section{RESULTS}

Prior to the process intervention, zero patients had a bedside evaluation prior to leaving the ICU. After implementation of the new process, $13.6 \%$ of patients transferred out of the ICU had a bedside evaluation completed and documented in the medical record by the ICU team prior to transfer. Debriefing with house staff in the ICU revealed barriers to compliance with this process. First, it was felt that too much time was required to complete the bedside evaluation, and that this detracted from the residents' ability to care for other critically ill patients or be present on rounds. Additionally, many transfers occurred overnight when the covering resident was less familiar with the patient's plan of care, and staffing ratios are reduced which exacerbated the time burden of this process.

At the beginning of the new structure, zero patients had their staff information changed by the receiving team. Following implementation of this process change, 50\% of patients had the updated physician staff information order placed by the receiving team, signifying that the floor team had received a verbal and written handoff prior to the patient leaving the ICU. Analysis of patient flow data before and after the pilot demonstrated no significant difference in time to move a patient once a bed had been assigned.

\section{DISCUSSION \& SYSTEMATIC BARRIERS TO IMPROVEMENT}

Handoffs are a critical component of quality care of the hospitalized patient, and require constant vigilance to maintain high quality given their increasing frequency. At our institution, handoffs from one unit to another within the hospital were identified on many levels to be an area needing improvement, and the HQSLC set out to demonstrate that standardization of the content and process of handoffs is crucial to improving the safety of our patients.

Although our pilot for a new transfer workflow resulted in only a modest behavior change in practice, it stimulated significant dialogue around handoff practice and culture. We believe this housestaff driven discussion helped to make safe handoffs an institutional priority. With this project occurring just prior to a transition to a new electronic medical record, our results informed the creation of a new electronic documentation workflow for patient flow from the ICU to the floor affecting lasting culture change at our institution.

With the proven validity of IPASS as a standardized method for effective handoffs, we were able to demonstrate to GME leadership at our institution the necessity to provide this training to all house staff. As a result, all incoming interns in 2017 were trained in IPASS using a curriculum that was developed with abbreviated IPASS materials specific to our hospital system. 
We were met with several large barriers implementing the ICU to floor handoff change. The extensive heterogeneity of handoffs in practice seemed to be the greatest barrier to improvement. Handoffs and transitions of care are by nature heterogeneous. Different providers require different subsets of information and have different priorities when giving and receiving handoffs. However, best practices have been described and the creation of a shared mental model, where all providers have the same expectation of content and process of a good handoff, is crucial to excellent patient care.

Conceptually, handoffs can either be viewed as black and white ("Patient is mine, and now he's yours"), or they may acknowledge a "grey period" of shared responsibility. Structures, such as closed units, and stressful working conditions reinforce a black/white mindset, and opportunities for collaboration to meet the patient's needs may be missed.

In addition, we found that leadership buy-in for improvement in handoff culture varied across departments and units. We found that driving change from below in this area is difficult without a mandate and support from faculty and senior house staff. Buy-in needs to be achieved at all levels of interprofessional practice, departmental and educational leadership, and hospital administration. Unfortunately, lack of support for process change can arise from various concerns, such as pressures to meet benchmarks for time to patient transfer, at the expense of good quality handoffs. In reality, the need for rapid and efficient transitions in care should make the role of clear, effective and standardized communication that much more vital.
Finally, a broad push for handoff education across the institution will help to create a common language and vocabulary for effective communication and higher quality handoffs. Training incoming and junior staff appears to be the most feasible approach to encourage and reinforce behavior change from the ground up, as was completed at our institution. To ensure continued lasting success in handoff safety initiatives at TJUH, the HQSLC has continued to disseminate IPASS education to senior residents across all GME training programs, with a total of nine programs trained to date. In order to evaluate the quality of handoffs, including the appropriate use of the IPASS format, HQSLC members are currently participating in formal interdisciplinary handoff observations involving real time, direct feedback. Further research efforts are necessary to continue to evaluate the effect of standardized handoff training in improving the perception of handoff safety at TJUH and its role in improving patient safety.

\section{REFERENCES}

1. Warrick D, Gonzalez-del-Rey J, Hall D, Statile A, White C, Simmons J, Wong SP. Improving resident handoffs for children transitioning from the intensive care unit. Hospital Pediatrics. 2015;5(3),127-133.

2. Aylward MJ, Rogers T, Duane PG. Inaccuracy in patient handoffs: Discrepancies between resident-generated reports and the medical record. Minnesota Medicine. 2001;94(12),38-41.

3. Starmer AJ, Spector ND, Srivastava R, et al. Changes in Medical Errors after Implementation of a Handoff Program. New England Journal of Medicine. 2014;371(19):1803-1812.

4. Starmer AJ, Spector ND, Srivastava R, et al. I-pass, A mnemonic to standardize verbal handoffs. Pediatrics. 2012;129(2),201-204.

5. Committee on Quality Health Care in America, Institute of Medicine. Crossing the Quality Chasm: A New Health System for the 21st Century. Washington, D.C.: National Academy Press; 2001. 